\title{
Functional Equivalence Applied in the Translation of Poems
}

\author{
Yu-Ting MA ${ }^{1, a,{ }^{*}}$ \\ ${ }^{1}$ Business School, Sichuan Agricultural University, China \\ a568550457@qq.com \\ ${ }^{*}$ Corresponding author
}

\begin{abstract}
Keywords: Functional Equivalence, Translation, Poems.
\end{abstract}
\begin{abstract}
As a translation theory, Nida's functional equivalence theory plays an important role in translation study. It guides us to the true nature and methods of translation. Poetry, a necessary genre in the field of literature, has been regarded as the very essence of art. Translation of this genre weighs a lot in translation study. Based on Nida's functional equivalence theory, this paper conducts a study on how to translate poems in different languages and cultures on phonetic level, lexical level, and syntactic level. Translation strategies applied are to be found on these levels.
\end{abstract}

\section{Introduction}

Functional equivalence is put forward by Nida, "the patriarch of translation study and a founder of the discipline" (Snell-Hornby 1988:1). This theory is regarded as his greatest contribution to translation. His proposal of this theory shows us a new way of translating in different cultures [1].

At the very beginning Nida calls this kind of translation as dynamic equivalent translation and his theory at this stage is called dynamic equivalence theory. In such a translation, one is not so concerned with matching the receptor-language message with the source language message, but with the dynamic relationship, that the relationship between receptor and message should be substantially the same as that which existed between the original receptors and the message (Nida 1964:159).

Then in 1986, in order to avoid ambiguousness, Nida used "functional equivalence" to replace "dynamic equivalence" functional equivalence concentrates on the concept of function. And it means that the reader of a translated text should be able to comprehend it to the point that they can conceive of how the original readers of the text must have understood and appreciated it. That is to say the effect of the text on readers in different cultures should be the same [2 4].

In order to produce functional equivalence some rules and principles are needed to follow by translators. if a translation is likely to arose misunderstandings of the meaning, certain changes and adjustments must be introduced into the text of the translation or the literal translation may be retained and a footnote explaining the likely misunderstandings must be added. secondly, if a close, formal translation makes no sense, i.e. it is totally obscure in designative meaning, certain changes may be introduced into the text unless the source text is purposely obscure; thirdly, if a close, formal translation is so semantically and syntactically difficult that people in target language are very likely to give up trying to understand it, certain changes are warranted, although it may be useful to indicate the nature of such changes in an introduction or in footnotes; fourthly, if a close, formal translation is likely to result in serious misunderstandings of the associative meanings of the source text or in a significant loss in a proper appreciation for the stylistic values of the source text, it is important to make such adjustments as are necessary to reflect the associative values of the source text; fifthly, the manner in which a translation is to be used has a significant influence upon the extent to which adjustments are to be made; at last, the fact that a source text must be translated in such a way as to occur with accompanying codes usually requires a number of adjustments on all levels: phonology, lexicon, syntax, and discourse. It requires a number of adjustments on all levels: phonology, lexicon, syntax, and so on [5].

We can infer from the above information that target readers' response play the most important rule in the process of translating. This paper shows how the theory of functional equivalence is applied in the translation of English poems into Chinese. 


\section{Application of Functional Equivalence in the Translation of Poems}

Poetry, as a special type of literature, plays a very important part in a country's culture. It is regarded as the most valuable legacy of a nation. As an aesthetic form of literature, poetry almost covers all the artistic features of all literature forms. The beauty of poetry lies in its phonetic musical rhythm, conciseness of words, various forms, abundance in meanings. However, all these bring about difficulties to the translation of poetry. Though the loss of meaning and aesthetic features is unavoidable during the process of translation, translation of poetry still weighs a lot in cultural communication. Due to lack of mutual knowledge of different cultures, some images and expressions cannot be understood by people from different languages and cultures. And all these become the obstacles for them to understand the poetry and for poetry to produce the same effects on different target readers. And Nida's functional equivalence theory provides us with a practical theoretical guidance to the translation of poems [6].

We all know that expression of feelings is the most obvious feature of poems. And poems have their characteristics on the level of phonetics, lexicon and syntax. All these levels play important roles in the expression of meaning. Based on these reasons, the effect of poems on the readers is the key point during the translation in different cultures. Functional equivalence is an ideal theory to guide the translation of poems in different cultures and languages.

On the phonetic level, rhyme can never escape translators' attention. It plays the most important role in the meaning expressing of poems, which convey and produce the feelings of the poets. Nida introduced isomorph into translation studies. He believes the expressions in different languages, which evoke similar response from the receptors, are functional isomorph, regardless of their forms. Nida proposed, "Translation is a process of picking out functional isomorph on various levels of different language. Translators need to look for the functionally equivalent words, grammar and rhetoric continuously." (Tan 2002:80) Poets usually express their feelings by various phones. Phonetic experience enables the readers to relate in mind some material features in the material world, when receiving different phones with all kinds of message and associations. By mere reading, the feelings of poets can be partially touched by readers through acoustic effects, even though the readers do not recognize the meaning of the specific language. In English, it has alliteration, internal rhyme and end rhyme. "When I am dead, my dearest, Sing no sad songs for me;" in these two lines, dead rhymes with dearest, and Sing rhymes with sad and songs. This kind of rhyme is called alliteration. But in Chinese, in most poems end rhyme occurs, internal rhyme and alliteration seldom appear in the poems. According to functional equivalence theory, in order to produce the same effect on the readers of different languages, some changes and adjustments are needed. If the translation is from English into Chinese, alliteration and internal rhyme may be replaced by end rhyme. If the translation is from Chinese into English, alliteration and internal rhyme occur.

On the lexical level, in order to emphasize the effects produced by normal word arrangements, some poems violate the normal rules of lexical usage. Meaning generation of any type of literature, to the most degree, relies on lexicon, especially poetry. In any poem, the words applied are limited, with each playing its unique role. There is no difference among words themselves, and even words in poetry are familiar to everyday use. The essence in poem writing lies not in the piling up of the most beautiful words to express feelings and produce effects, but in the organization of words beautifully and skillfully by poets and how one word goes with others. We can see that the appropriate use of words isn't decided by the words themselves, but by the collocations with others. Some poets can make totally irrelevant words go together, and profound sense and effects can be created. Obsolete words and expressions are always abandoned, and new expressions are created to replace them. Poetry is an art of enrichment. A good poem uses the fewest words to express the most profound meanings and feelings. In English, a word possesses other different connotations and implications besides its fundamental meaning. This is what we call polysemy, which provides poets with convenience to generate various and profound meanings and unexpected effects with fewer words. However, the meaning of a word is not fixed, but rather affected by context and the words before and after it. Poets always make the most use of it, making subtle difference of a word to introduce a world of difference. Poem itself is a special language, and the meaning of a word differs in the eyes of 
receptor sharing different culture and education background. Sometimes a verb occurs at the place where a noun or an adjective is needed, or a word that illustrates the properties of human beings is used to describe an animal or other irrelevant things. In some cases, an abstract word occurs where a concrete word is expected or a concrete word occurs where an abstract word is expected to make the acceptors experience the real and imaginary worlds. It arouses a kind of feeling that the real and imaginary worlds are an integral part, and no difference exists between the two. Maybe they are the same, and there is no need to separate the imaginary world from the real world. However, it sometimes confuses the poet about what is real or what is imaginary. In the process of translation, if the same effect on readers in different language is to achieve, some adjustments must be made.

Syntax, the structure of words, phrases, clauses, and sentences, is one of the major determinants of poetic expressions. With its complex constraints on well-form, syntax is a major locus of linguistic creativity. Most metaphors begin with some kind of syntactic deviance which has strong effects on poet's expression of feelings and reader's perception of the meaning. Syntax can also be used as a powerful icon and its structure can be extended, repeated, and so forth [7]. What we want to see is their poetic values here. In poetry, in order to realize poetic functions and artistic functions, the poet inclines to adopt deviant sentences. A different type of syntactic principle is formed by violating regular syntax. In order o achieve harmonious rhymes, to express poet's inner feelings, to convey experience, to leave space for imagination and thoughts, inverted sentences are often used to replace the regular sentences. Reasonable inverted sentences introduce good rhetoric effects, which is effective in realizing the artistic representation of poems and in producing aesthetic effects. Some poets are mostly eccentric and the oddities in syntax in their poems represent their conceptual eccentricity and oddity. Their thoughts and feelings are odd and out of ordinary way, which is in accordance with their poetry, conveying them with extraordinary strength and methods. In the ordinary way, nonsense and eccentric syntax may not make their sense, however, in poetry, that's the other way round, they produce great poetic effects. Some poets apply severe revisions of transformational rules and tries to disintegrating meaning, which makes the readers come closer to their thoughts the inference triggers. And violated and deviant syntax contributes greatly to the identity of the poets. Things or images in their poems that arouse a cloying oddness draw attention to them, show a strained novelty and achieve great profundity. In some of her poems, oddity is achieved for the lack of cohesion and coherence, expressing the disorder in the material world, realizing harmony deep in it. In some poems, the syntactic and lexical economy occurs everywhere by using ellipsis or moving certain expressions and elements away from the ordinary and natural constituents. Meaning expressed in this way is often implied by the lines of inference and the entire context. However, the elimination in the poems gains a lot in effect; it also imposes serious impairment to the possibilities of interpretation [8]. To increase the degree of uncertainty and processing effort, poets create a problem for the translators to deal with, that they have to decide whether to maintain a syntactic eccentricity or to produce a more comprehensible poem, under the circumstance that readers speaking different languages have the same response to the poem. And the syntax in poets can be categorized into three types: paratactic syntax, hypotactic syntax and negation and contrast pattern. In the process of translation, if the same effect on readers in different language is to achieve, some adjustments must be made.

\section{Conclusion}

On phonetic level, during the process of translating poems, adjustments and changes are needed to realize the same aesthetic effects. In order to transfer the meaning clearly, rhyme weighs a lot. As we all know, rhyme is the very key element in forming the essence of a poem, if translation without taking it into consideration, there must be tremendous loss on the perceiving of meaning.

On lexical level, choosing the proper single word in the poem is important. While taking context into consideration, we strive to find good ways to solve the problems existing in translation and to get closer to the poet. 
On syntactic level, the characteristics embedded in the original syntax are maintained during the process of translating. According to functional equivalence theory, it can be described as enabling access to the same response which the original produces in its source language and culture.

\section{References}

[1] Johnson, Thomas H. ed. The Complete Poems of Emily Dickinson. Kent: Mackays of Chatham PLC, 1975.

[2] Katan, David. Translating Cultures: An Introduction for Translators, Interpreters and Mediators, Shanghai: Foreign Language Education Press, 2004.

[3] Landers, Clifford E. Literary Translation: A Practical Guide. Shanghai: Foreign Language Education Press, 2008.

[4] Leech, Geoffrey N. A linguistic Guide to English poetry. Beijing: Foreign Language Teaching and Research Press, 2001.

[5] Lefevere, Andre. Translating Literature: Practice and Theory in A Comparative Literature

[6] Newmark, Peter. A Textbook of Translation. Shanghai: Shanghai Foreign Language Education Press, 2001.

[7] Nida, Eugene A. Language and Culture: Contexts in Translating. Shanghai: Shanghai Foreign Language Education Press, 2001.

[8] Wilss, W. The Science of Translation: Problems and Methods Shanghai: Shanghai Foreign Language Education Press, 2001. 